\title{
A general approach to obtain soft x-ray transparency for thin films grown on bulk substrates
}

Manuel Fohler, Stefanie Frömmel, Michael Schneider, Bastian Pfau, Christian M. Günther, Martin Hennecke, Erik Guehrs, Laura Shemilt, Durgamadhab Mishra, Dirk Berger, Sören Selve, Dmitriy Mitin, Manfred Albrecht, and Stefan Eisebitt

Citation: Review of Scientific Instruments 88, 103701 (2017); doi: 10.1063/1.5006522

View online: $h t t p: / / d x . d o i . o r g / 10.1063 / 1.5006522$

View Table of Contents: http://aip.scitation.org/toc/rsi/88/10

Published by the American Institute of Physics

\section{Articles you may be interested in}

Note: A modified optics based technique for suppressing spurious signals in photoreflectance spectra

Review of Scientific Instruments 88, 106103 (2017); 10.1063/1.4993263

A platform for exploding wires in different media

Review of Scientific Instruments 88, 103504 (2017); 10.1063/1.4996027

Hardware for dynamic quantum computing

Review of Scientific Instruments 88, 104703 (2017); 10.1063/1.5006525

X-ray spectrometer having 12000 resolving power at $8 \mathrm{keV}$ energy

Review of Scientific Instruments 88, 103107 (2017); 10.1063/1.4999995

Ring-averaged ion velocity distribution function probe for laboratory magnetized plasma experiment

Review of Scientific Instruments 88, 103507 (2017); 10.1063/1.4986589

Evaluation of low-frequency operational limit of proposed ITER low-field-side reflectometer waveguide run including miter bends

Review of Scientific Instruments 88, 103508 (2017); 10.1063/1.4995662

\section{CERN pays the APC}

Now CERN-funded researchers can publish their methods articles open access in EPJ Techniques \& Instrumentation, and CERN is sponsoring article-processing charges (APCs)! Details here.

\section{Springer}

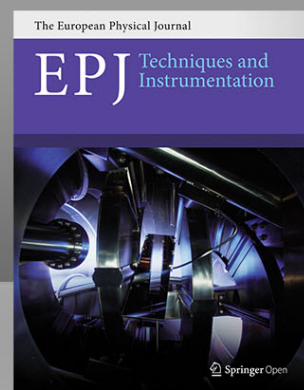




\title{
A general approach to obtain soft x-ray transparency for thin films grown on bulk substrates
}

\author{
Manuel Fohler, ${ }^{1,2, a)}$ Stefanie Frömmel, ${ }^{1, a)}$ Michael Schneider, ${ }^{1,3}$ Bastian Pfau, ${ }^{3}$ Christian \\ M. Günther, ${ }^{1,3}$ Martin Hennecke, ${ }^{3}$ Erik Guehrs, ${ }^{1}$ Laura Shemilt, ${ }^{1}$ Durgamadhab Mishra, ${ }^{2}$ \\ Dirk Berger ${ }^{4}$ Sören Selve, ${ }^{4}$ Dmitriy Mitin, ${ }^{5}$ Manfred Albrecht, ${ }^{5}$ and Stefan Eisebitt ${ }^{1,3, b)}$ \\ ${ }^{1}$ Institut für Optik und Atomare Physik, Technische Universität Berlin, Straße des 17. Juni 135, 10623 Berlin, \\ Germany \\ ${ }^{2}$ Helmholtz-Zentrum Berlin für Materialien und Energie GmbH, Albert-Einstein-Str. 15, 12489 Berlin, Germany \\ ${ }^{3}$ Max-Born-Institut für Nichtlineare Optik und Kurzzeitspektroskopie, Max-Born-Straße 2A, 12489 Berlin, \\ Germany \\ ${ }^{4}$ Zentraleinrichtung Elektronenmikroskopie, Technische Universität Berlin, Straße des 17. Juni 135, 10623 Berlin, \\ Germany \\ ${ }^{5}$ Institut für Physik, Experimentalphysik IV, Universität Augsburg, Universitätsstraße 1 Nord, 86159 Augsburg, \\ Germany
}

(Received 4 May 2017; accepted 24 September 2017; published online 19 October 2017)

\begin{abstract}
We present a general approach to thin bulk samples to transparency for experiments in the soft $\mathrm{x}$-ray and extreme ultraviolet spectral range. The method relies on mechanical grinding followed by focused-ion-beam milling. It results in a uniformly thin area of high surface quality, suitable for nanoscale imaging in transmission. In a proof-of-principle experiment, nanoscale magnetic bits on a commercial hard drive glass disk are imaged with a spatial resolution below $30 \mathrm{~nm}$ by soft $\mathrm{x}$-ray spectro-holography. Furthermore, we demonstrate imaging of a lithographically patterned test object via absorption contrast. Our approach is suitable for both amorphous and crystalline substrates and has been tested for a variety of common epitaxy growth substrates. Lateral thinning areas in excess of $100 \mu \mathrm{m}^{2}$ and a remaining substrate thickness as thin as $150 \mathrm{~nm}$ are easily achievable. Our approach allows preserving a previously grown thin film, and from nanofocus electron diffraction, we find no evidence for morphological changes induced by the process, in agreement with numerical simulations of the ion implantation depth distributon. We expect our method to be widely applicable and especially useful for nanoscale imaging of epitaxial thin films. Published by AIP Publishing. https://doi.org/10.1063/1.5006522
\end{abstract}

\section{INTRODUCTION}

Nanoscale spectro-microscopy utilizing soft x-rays has evolved to an important tool in nanoscience for the investigation of samples with structure size or inhomogeneity down to the $10 \mathrm{~nm}$ level. The use of soft x-rays with wavelengths between $10 \mathrm{~nm}$ and $1 \mathrm{~nm}$ (i.e., roughly between $100 \mathrm{eV}$ and $1000 \mathrm{eV}$ photon energy) is of particular importance as they provide unique spectral information due to comparably small lifetime widths of the accessible core levels, resulting in the possibility to obtain spectroscopic contrast based on atomic species, chemical as well as magnetic state, and topography. To obtain such contrast, the photon energy cannot be chosen at will but must be tuned to be in resonance with a suitable electronic transition, which for all light elements and many useful resonances of heavier elements is in the soft $\mathrm{x}$-ray domain. Both full-field and raster imaging techniques are frequently used in the transmission mode, due to (i) the possibility to probe buried structures via the combination of bulk sensitivity and atomic selectivity, (ii) the photon-in photon-out advantage of being able to measure in applied

\footnotetext{
a)M. Fohler and S. Frömmel contributed equally to this work.

b)Author to whom correspondence should be addressed: eisebitt@mbiberlin.de
}

electrical or magnetic fields and without detrimental effects associated with photoemission-induced charging, and (iii) the technical advantages of operating symmetrically around the optical axis, allowing for high spatial resolution in full-field approaches. Examples for such soft x-ray transmission techniques with spatial resolutions approaching approximately $10 \mathrm{~nm}$ are Transmission X-ray Microscopy (TXM), ${ }^{1,2}$ Scanning Transmission X-ray Microscopy (STXM), ${ }^{3}$ Fourier Transform X-ray Holography (FTH) ${ }^{4}$ and Coherent Diffractive Imaging (CDI). ${ }^{5}$ As the exit wave of the incident radiation after passage through the sample is detected, all these approaches require suitable soft x-ray transparency of the sample to be investigated. Depending on the material and the wavelength used, the attenuation length is typically found to be between $20 \mathrm{~nm}$ and $1 \mu \mathrm{m}$ in the soft x-ray regime.

This requirement has severely hampered nanoscale imaging and spectroscopy for many material classes in the past. For example, the vast material class of epitaxial thin films has not been accessible in transmission (spectro-)microscopy. The lattice constants of the film to be grown dictate the use of suitably matched growth crystals which are generally macroscopic in thickness and hence opaque to soft $\mathrm{x}$-rays. Photoemission electron microscopy (PEEM) with soft $\mathrm{x}$-ray excitation has been able to fill this gap for such systems where surface sensitivity is advantageous ${ }^{6}$ but also comes with restrictions regarding 
suitable samples and sample environments. Of particular interest are complex oxides and materials with strong electronic correlation, where unique functionalities such as low temperature superconductivity or colossal magnetoresistance exist. Here, epitaxial growth is mandatory to control the material properties as well as possible. At the same time, due to the complex phase diagram with competing phases, nanoscale inhomogeneity in the form of domain formation and phase separation is suspected to crucially influence the macroscopic properties. For the special case of perovskite membranes, the production of free-standing films via sacrificial water-soluble layers has recently been reported, illustrating the importance of obtaining access to epitaxial thin films in transmission experiments. $^{7}$

Here, we report on a general thinning approach, applicable to samples grown on both crystalline and amorphous bulk substrates with nanoscale soft $\mathrm{x}$-ray imaging applications in mind, where transparency is required over a lateral extent of a few micrometers. In a proof-of-principle experiment, we demonstrate the suitability for transmission imaging with X-ray Spectro-Holography, ${ }^{8}$ focusing on thin magnetic films. However, the proposed preparation method is also capable of producing larger membrane areas as required in Soft X-ray Transmission Microscopy, Soft X-ray Spectroscopy, ${ }^{9}$ and Small-Angle X-ray Scattering (SAXS). ${ }^{10}$

\section{SAMPLE PREPARATION METHOD}

The thinning procedure combines a two-step wet grinding technique, adapted from Transmission Electron Microscopy (TEM) plan view specimen preparation, ${ }^{11,12}$ with custom Focused-Ion-Beam (FIB) milling. ${ }^{13}$ In Fig. 1, we illustrate the processing steps of our preparation technique. In the first step, the thin film to be ultimately investigated is protected during the thinning procedure via coating with a PMMA film of about $400 \mathrm{~nm}$ thickness. After the mechanical thinning procedure, this film will be removed by dissolution in acetone. The protective PMMA layer is robust enough to allow the fixing of the sample with the growth side carrying the thin film to a standard glass cylinder [Fig. 1(a)] using a thermoplastic polymer (Crystalbond, $120^{\circ} \mathrm{C}$ ). Disk grinding with a lubricant containing $15 \mu \mathrm{m}$ diamond abrasive particles is used to reduce the substrate thickness to around $80 \mu \mathrm{m}$ [Fig. 1(b)]. Next, dimple grinding with a flat bronze wheel and felt wheel, using 3 $\mu \mathrm{m}$ and $1 \mu \mathrm{m}$ diamond lubricants, respectively, under a contact pressure of $20 \mathrm{~g}$ is employed to thin the sample down to a remaining $8 \mu \mathrm{m}$ thickness at the lowest point of the dimple [Fig. 1(c)]. An optical microscope is used to check the sample thickness. The sample roughness is reduced using alumina polishing suspension on a fresh felt wheel for around $15 \mathrm{~min}$ utes. To reduce mechanical tension on the thin sample region, a silicon frame surrounding the dimple region is glued to the substrate side of the sample prior to separating the specimen from the glass cylinder by acetone [Fig. 1(d)]. Depending on the compatibility of the thin film growth process with such a pre-thinned substrate, the thin film growth can also be carried out at this stage.

Thinning to soft $\mathrm{x}$-ray transparency is carried out with $30 \mathrm{kV}$ focused $\mathrm{Ga}^{+}$ions under Scanning Electron Microscopy (SEM) control in a FEI Helios NanoLab 600 dual beam instrument. To avoid charging problems in insulating or badly conducting substrates deteriorating both FIB patterning resolution and SEM process control, a thin conductive layer (e.g., $\mathrm{Cr}$ or amorphous carbon) is deposited on the dimpled sample surface prior to FIB milling, reducing electron transport paths between this conductive layer and the $\mathrm{Ga}^{+}$interaction region to the micron scale. FIB milling can then be used to thin the substrate to a desired remaining thickness below $1 \mu \mathrm{m}$ over a lateral extent easily in excess of $100 \mu \mathrm{m}^{2}$ [Fig. 1(e)]. The desired membrane thickness is achieved by both prior milling of test areas and measuring the SEM contrast change between the thinned area and surrounding substrate in SEM images of the film side opposite to the dimple. At this stage, the thinned sample can be used for a variety of soft x-ray experiments in transmission, including time-resolved spectroscopy as well as spectro-microscopic imaging.

\section{PREPARATION OF SAMPLES AND EXPERIMENTAL DETAILS}

We demonstrate the approach using spectro-holography with soft $\mathrm{X}$-rays in order to image magnetic domains in a

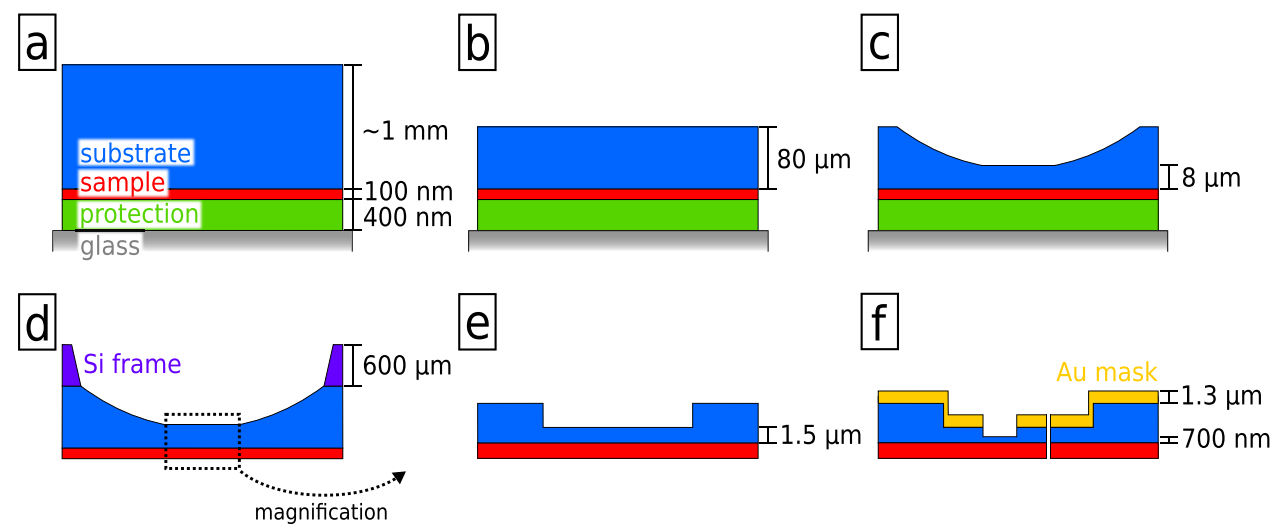

FIG. 1. Schematic illustration of the steps involved in the sample preparation process. (a) Growth substrate (blue) with thin film layer of interest (red). The thin film layer is protected by a PMMA layer (green) before the sample preparation holder (gray) is applied. (b) Substrate back-thinned by plane disk grinding and (c) dimple grinding. (d) A silicon frame (purple) surrounding the dimple area is applied to increase mechanical stability before the sample holder is removed. (e) Using FIB milling, the substrate is thinned down further to a membrane area. (f) A Cr/Au multilayer coating (yellow) is applied to be used for the FTH mask with an object hole and a reference through hole. The object area is thinned down further to the final substrate thickness. 
commercial magnetic data storage medium via a transmission measurement using X-ray Magnetic Circular Dichroism (XMCD) contrast at the $\mathrm{Co}_{3}$ absorption edge. The sample is a piece of an off-the-shelf $2.5 \mathrm{in}$. HGST Travelstar Z5K500 family hard disk drive consisting of a glass disk of $642 \mu \mathrm{m}$ thickness coated with a CoCrPt-based thin film stack which can be assumed to be similar to perpendicular recording media described by Piramanayagam et al. ${ }^{14}$ A magnetization test pattern was written using a conventional read/write head of a Scanning Magnetoresistive Microscope (SMRM). ${ }^{15}$ The pattern consisted of magnetization transitions with pitch sizes continuously varying from $30 \mathrm{~nm}$ to $210 \mathrm{~nm}$ in the $y$-direction (down-track), which were repeated over an area of about $100 \times$ $100 \mu \mathrm{m}^{2}$.

To define a Fourier Transform X-ray Holography (FTH) geometry, $8,16,17$ the final FIB thinning step was interrupted when the sample had $1.5 \mu \mathrm{m}$ thickness and a soft x-ray mask consisting of a $\mathrm{Cr} / \mathrm{Au}$ multilayer with $1.3 \mu \mathrm{m}$ thickness was deposited. Subsequently, circular object apertures with $1.5 \mu \mathrm{m}$ diameter were patterned, with a target value of $700 \mathrm{~nm}$ remaining glass substrate thickness, corresponding to approximately $50 \%$ transmission at $1.59 \mathrm{~nm}$ wavelength. Use of soft x-rays at this wavelength is required to obtain XMCD contrast at the Co $\mathrm{L}_{3}$ absorption edge in order to image the test pattern. Finally, apertures to define the reference beam for holography were written as through holes in the vicinity of the object apertures [Fig. 1(f)].

Spectro-holographic imaging of this sample was carried out at the P04 beamline at the PETRA III synchrotron source. The hologram was recorded on a 4M-pixel in-vacuum backilluminated charge-coupled device (CCD) camera. Blocking the intense radiation on the optical axis with a central beamstop allowed us to record the hologram modulations to high momentum transfer despite the CCD's limited dynamic range. Holograms were recorded with positive and negative circular polarization, each 100 accumulations of $0.95 \mathrm{sec}$ exposures, with the difference of the polarizations carrying the magnetic information. ${ }^{8}$ The reconstruction of the real-space image was obtained via a 2D Fourier transform of the hologram and free-wave propagation along the optical axis to the thin film sample plane. ${ }^{18,19}$

In the second test, utilizing absorption contrast rather than $\mathrm{XMCD}$, a gecko-shaped test pattern was deposited on a crystalline $\mathrm{MgO}(100)$ substrate using electron-beam induced $\mathrm{Pt}$ deposition from organic precursor molecules. Due to the deposition process, the approximately $50 \mathrm{~nm}$ thick test object is estimated to consist of only $20-50$ at. $\% \mathrm{Pt}$, with the remainder being carbon from the precursor molecules. ${ }^{20}$ After the mechanical grinding process, FIB thinning was used to produce a thinned area of $30 \times 30 \mu \mathrm{m}^{2}$ with a remaining thickness of around $200 \mathrm{~nm}$ before the test pattern was deposited. Here, the object hole in the $1.3 \mu \mathrm{m}$ thick $\mathrm{Cr} / \mathrm{Au}$ multilayer defining the field of view had a diameter of $3 \mu \mathrm{m}$. Holographic imaging was carried out at the U41-PGM beamline of the BESSY II synchrotron source at $1.75 \mathrm{~nm}$ wavelength.

We have tested the applicability of our approach for various commonly used crystalline growth substrates. With the mechanical grinding steps using diamond abrasive particles being almost universally applicable for crystals, we focus on the possibility to perform the crucial final thinning via FIB milling. We compare the results of material removal on $\mathrm{SrTiO}_{3}(100), \mathrm{Si}(100), \mathrm{MgAl}_{2} \mathrm{O}_{4}(100), \mathrm{Al}_{2} \mathrm{O}_{3}(0001), \mathrm{Gd}_{3} \mathrm{Ga}_{5}$ $\mathrm{O}_{12}(111)$, and $\mathrm{LiF}(100)$ on a $20 \times 20 \mu \mathrm{m}^{2}$ test area. Thinning is carried out via FIB milling with gallium ions in the FIB/SEM dual beam instrument. Again, a thin conducting layer of carbon was deposited on the samples prior to FIB milling. $\mathrm{A} \mathrm{Ga}^{+}$ current of $19 \mathrm{nA}$ at $8 \mathrm{kV}$ acceleration voltage has been used with the same total dose per area of $23.2 \mathrm{nC} / \mu^{2}$ for each sample, which would equivalently allow us to mill approximately $40 \mu \mathrm{m}$ deep into polycrystalline gold. SEM images of the resulting areas have been taken to check milling uniformity and measure the resulting depths.

Especially for epitaxially grown samples, potential damage of the crystal structure of the thin film or the adjacent substrate layers during the final FIB thinning step is a concern. We have investigated such potentially detrimental effects in a magnetite $\left(\mathrm{Fe}_{3} \mathrm{O}_{4}\right)$ film of $71 \mathrm{~nm}$ thickness, grown via molecular beam epitaxy on a $\mathrm{SrTiO}_{3}(100)$ substrate (in the following abbreviated as STO). In this case, the mechanical grinding steps were carried out prior to MBE growth while all FIB milling steps were performed after the magnetite film had been grown on the substrate. The STO substrate was thinned to a final thickness of $150 \mathrm{~nm}$ over an area of $40 \times 40 \mu \mathrm{m}^{2}$, again using $30 \mathrm{kV} \mathrm{Ga}{ }^{+}$ions. Simulations of the $\mathrm{Ga}^{+}$depth distribution after implantation using the SRIM software package ${ }^{21}$ neglecting channeling effects, predict that $\mathrm{Ga}^{+}$ions after $30 \mathrm{kV}$ acceleration are deposited in a range up to $17 \mathrm{~nm}$, suggesting that thinning to remaining substrate thicknesses of $100 \mathrm{~nm}$ should be possible without deteriorating the crystal structure in the region of relevance. To analyze the crystallinity of the film, electron diffraction was carried out on a FIB-generated cross-sectional lamella of the magnetite film grown on STO, using a $200 \mathrm{keV}$ beam of the TEM.

\section{RESULTS AND DISCUSSION}

In Fig. 2, we present a comparison of the magnetic domain test pattern obtained via SMRM imaging prior to the thinning procedure [Fig. 2(a)] and as obtained via FTH after the procedure as described above [Fig. 2(b)]. Note that the representative area shown in the SMRM image is not necessarily identical to the field of view in the FTH image. The FTH

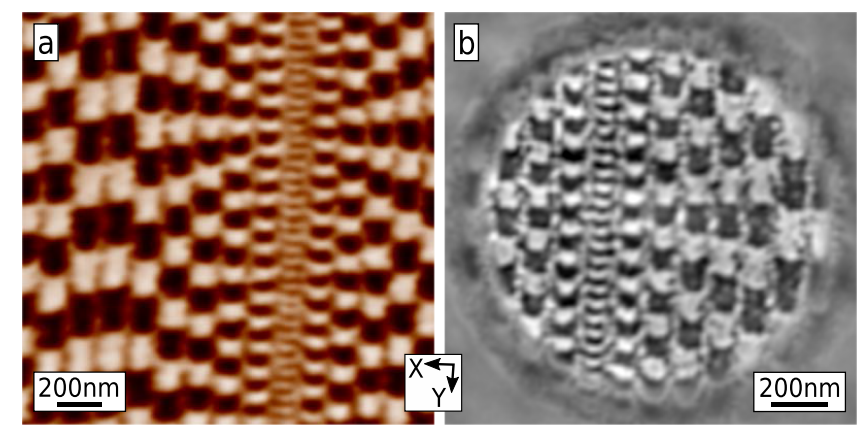

FIG. 2. Comparative magnetization images of the test pattern written on a conventional HDD medium obtained via (a) SMRM, prior to back-thinning preparation and (b) reconstruction of the soft x-ray FTH measurement on the back-thinned sample. 


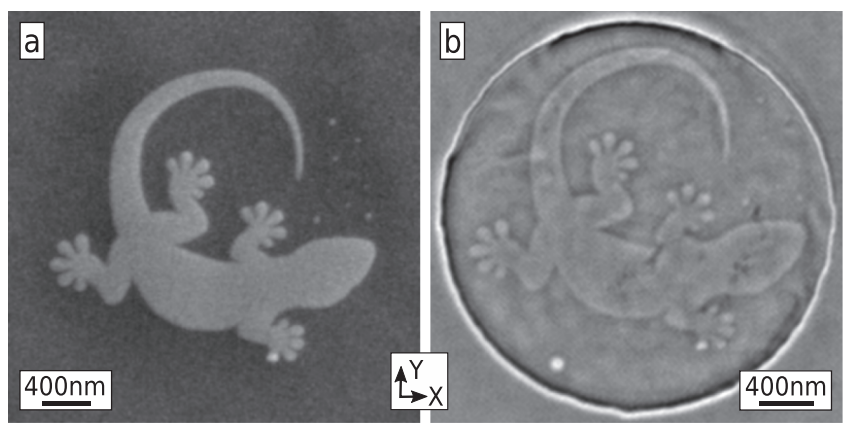

FIG. 3. Images of a gecko-shaped absorption test pattern obtained via (a) SEM imaging and (b) reconstruction of the soft x-ray FTH measurement. The circular aperture in the Au/Cr layer defining the field of view for FTH is on the opposite side of the sample and hence not visible in the SEM image.

reconstruction clearly reproduces the regular bit pattern in all detail down to the smallest pitch of $30 \mathrm{~nm}$. Furthermore, the $\mathrm{x}$-ray holography image allows us to discern curved bit boundaries caused by the write head field shape ${ }^{15}$ as well as intra-bit magnetic structures such as small areas pinned in the opposite magnetization direction. These details are barely visible in the SMRM images due to (i) the elongated size of the tunnel magnetoresistive sensor in the cross-track $(\mathrm{x})$ direction and (ii) the fact that the SMRM detects the magnetic stray field above the sample. In contrast, FTH in transmission geometry measures the projection of local magnetization of the entire depth of the magnetic film. The results show that the bit pattern was not altered during the thinning procedure and that high resolution transmission imaging with sub- $30 \mathrm{~nm}$ resolution through the thinned substrate is possible with our approach.

Results from the Pt/C test pattern are shown in Fig. 3, comparing the SEM image to the reconstruction from FTH. While SEM shows sample topography as reflected in the detected secondary electron yield, the transmission hologram reflects the absorption through the entire sample but is inherently high-pass filtered due to the use of a central beam stop when recording the hologram. This could be avoided in future experiments via a detector with a higher dynamic range or by extending the dynamic range via composite
TABLE I. Resulting milling depth and depth profile, obtained from SEM images, listed with the corresponding crystals bandgap energy (experimental values) for common epitaxy growth substrates.

\begin{tabular}{lccccc}
\hline \hline Figures & Material & $\begin{array}{c}\text { Crystal } \\
\text { orientation }\end{array}$ & $\begin{array}{c}\text { Bandgap } \\
(\mathrm{eV})\end{array}$ & $\begin{array}{c}\text { Milling } \\
\text { depth }(\mu \mathrm{m})\end{array}$ & Depth profile \\
\hline 4(a) & $\mathrm{SrTiO}_{3}$ & $(100)$ & 3.25 & 4.6 & Homogeneous \\
$4(\mathrm{~b})$ & $\mathrm{Si}$ & $(100)$ & 1.12 & 8.1 & Homogeneous \\
4(c) & $\mathrm{MgAl}_{2} \mathrm{O}_{4}$ & $(100)$ & 7.8 & 3.0 & Homogeneous \\
4(d) & $\mathrm{Al}_{2} \mathrm{O}_{3}$ & $(0001)$ & 7.6 & 2.5 & Homogeneous \\
4(e) & $\mathrm{Gd}_{3} \mathrm{Ga}_{5} \mathrm{O}_{12}$ & $(111)$ & 5.66 & 6.5 & Homogeneous \\
4(f) & $\mathrm{LiF}^{(\mathrm{f} F}$ & $(100)$ & 13.6 & 1.2 & Inhomogeneous \\
\hline \hline
\end{tabular}

holograms. The image of the object via FTH reveals all details of the test pattern down to the smallest feature sizes of $70 \mathrm{~nm}$.

In Table I, we list the milling uniformity and resulting depths for the crystal FIB thinning comparison. For all tested crystals with the exception of LiF, an even material removal is controllably achieved (Fig. 4). We expect charging effects due to the exceptionally large bandgap of $13.6 \mathrm{eV}$ in $\mathrm{LiF}$ to be the reason for the inhomogeneous milling behavior observed in this case. This means the important final FIB thinning step will work with most common crystalline substrates.

In Fig. 5(a), we present a TEM dark-field image of the FIB-generated cross-sectional lamella of the magnetite film grown on STO, with the probe beam for electron diffraction covering the magnetite film and the adjacent substrate as indicated. The resulting diffraction pattern is shown in Fig. 5(b). We observe a pattern clearly corresponding to a predominantly monocrystalline structure of both the magnetite film and the STO substrate, where the distinct reflections can be easily indexed. A small displacement of the STO and $\mathrm{Fe}_{3} \mathrm{O}_{4}$ peaks in reciprocal space reflects the lattice mismatch between both materials. The dislocations caused by this mismatch show up as grainy structure in the dark-field TEM image. Obviously, the crystallinity of the sample layer has not been destroyed during the FIB processing steps, as expected from the SRIM simulations.
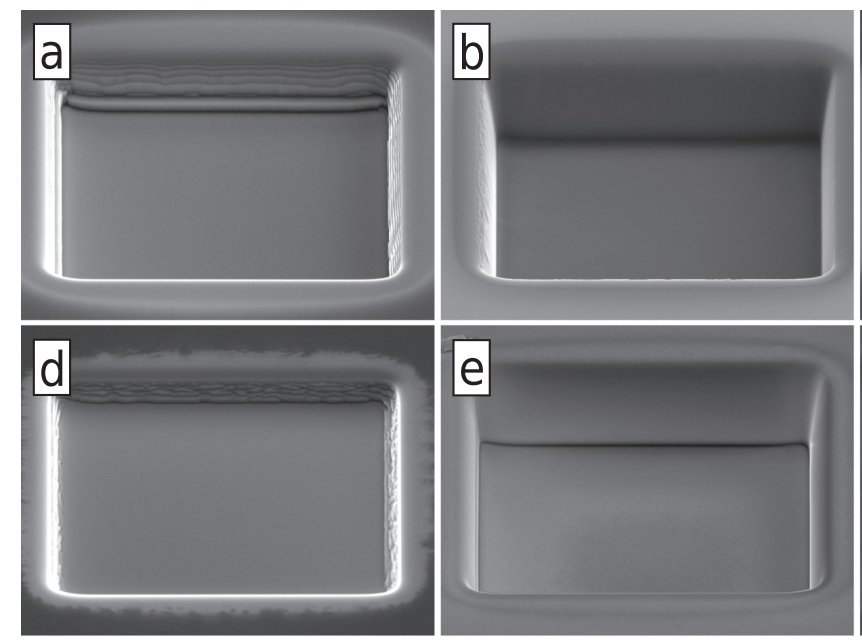

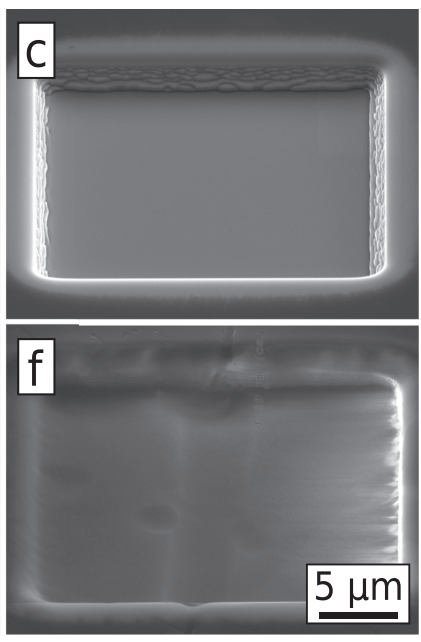

FIG. 4. SEM images of FIB substrate thinning test for (a) $\mathrm{SrTiO}_{3}(100)$, (b) $\mathrm{Si}(100)$, (c) $\mathrm{MgAl}_{2} \mathrm{O}_{4}(100)$, (d) $\mathrm{Al}_{2} \mathrm{O}_{3}(0001)$, (e) $\mathrm{Gd}_{3} \mathrm{Ga}_{5} \mathrm{O}_{12}(111)$, and (f) $\mathrm{LiF}(100)$. All crystals tested allow for smooth FIB milling, with $\mathrm{LiF}$ being the only exception. SEM images are recorded with a viewing angle tilted $52^{\circ}$ with respect to the sample normal, depth measurements listed in Table I have been corrected for this angle. 


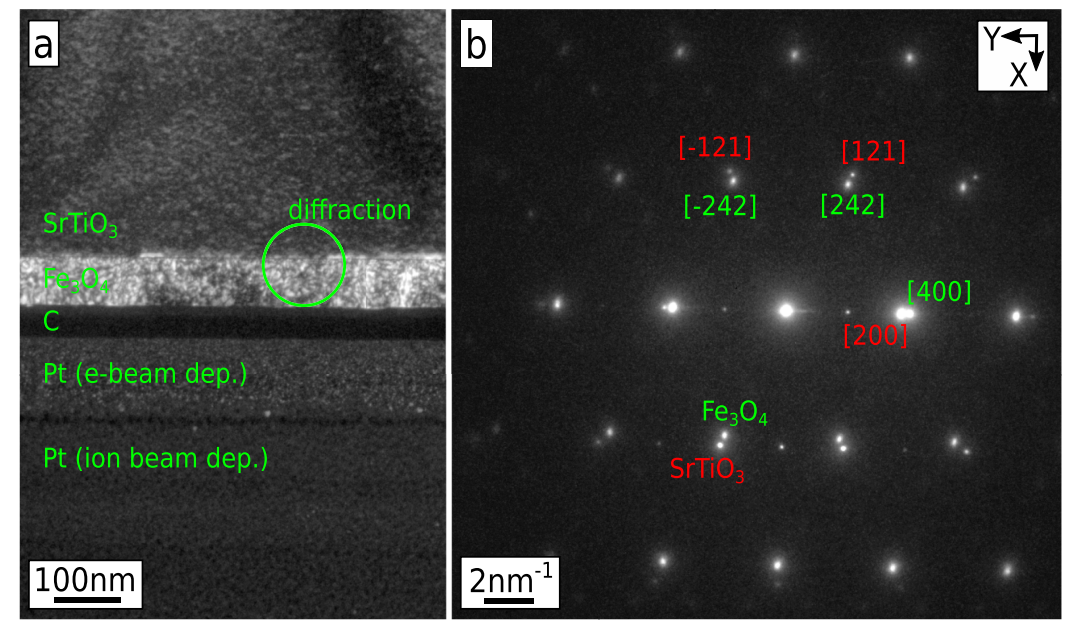

FIG. 5. (a) TEM dark-field image of the TEM lamella, representing a cross section of the $\mathrm{STO} / \mathrm{Fe}_{3} \mathrm{O}_{4}$ sample. The circle indicates the electron beam excited region for diffraction imaging. (b) Electron diffraction patterns of STO (red) and $\mathrm{Fe}_{3} \mathrm{O}_{4}$ (green), revealing the lattice mismatch between the substrate and the film. Position and shape of diffraction peaks indicate that both materials are monocrystalline.

In summary, we have demonstrated a widely applicable method to thin crystals and other macroscopic growth substrates to soft x-ray transparency, based on a combination of mechanical and ion beam thinning. Our approach does allow us to carry out soft x-ray spectroscopy and imaging experiments on thin epitaxial films in transmission, which have so far not been generally accessible by bulk sensitive spectromicroscopy techniques. We demonstrate that the proposed method works for a variety of commonly used crystals for epitaxial growth and show that thinning down to $150 \mathrm{~nm}$ substrate thickness is feasible without detectable damage in the crystallinity of the thin film or the adjacent growth substrate. Apart from the study of epitaxial thin films, our procedure also facilitates the investigation of functional nanoscale structures as found in many application environments, as demonstrated by high resolution holographic imaging of magnetic bits written on a commercial hard disk drive. Note that for such amorphous substrates, anisotropic etching approaches are not a patterning alternative. We expect the suggested technique to be of particular use in the study of nanoscale domain formation and phase separation in complex materials. This includes in particular oxidic materials, where on one hand, macroscopic properties are suspected to be linked to nano- and meso-scale ordering phenomena, ${ }^{22-25}$ while on the other hand, bulk information is desirable due to common stoichiometry variation in the topmost atomic layers. We would like to point out that the study of dynamic phenomena on the nanoscale such as the formation of transient phases ${ }^{26,27}$ or non-local phenomena mediated, e.g. by hot electron transport, ${ }^{28-30}$ will be greatly facilitated by our approach as it is fully compatible with the use of femtosecond soft $\mathrm{x}$-ray pulses at free-electron lasers or high harmonic generation sources. ${ }^{31-33} \mathrm{As}$, in particular, high harmonic sources are becoming much more widespread and progress from the extreme ultraviolet into the soft $x$-ray regime, ${ }^{34,35}$ we expect the proposed procedure to facilitate a variety of investigations of nanomaterials in the near future.

\section{ACKNOWLEDGMENTS}

We kindly thank P. Graus (Universität Kostanz, Konstanz, Germany) for his help with laser marking and A. E. Gafarov
(National Technical University of Ukraine, Kiev Polytechnic Institute, Kiev, Ukraine) for technical assistance. Furthermore, many thanks to S. Stöwer (Technische Universität Berlin, Institut für Optik und Atomare Physik, Berlin, Germany) for her assistance in the TEM preparation lab. We thank the DESY P04 beamline team for their excellent support.

${ }^{1}$ K. Henzler, P. Guttmann, Y. Lu, F. Polzer, G. Schneider, and M. Ballauff, Nano Lett. 13, 824 (2013).

${ }^{2}$ K. Henzler, A. Heilemann, J. Kneer, P. Guttmann, H. Jia, E. Bartsch, Y. Lu, and S. Palzer, Sci. Rep. 5, 17729 (2015).

${ }^{3}$ P. Guttmann and C. Bittencourt, Beilstein J. Nanotechnol. 6, 595 (2015).

${ }^{4}$ B. Pfau, C. M. Günther, E. Guehrs, T. Hauet, T. Hennen, S. Eisebitt, and O. Hellwig, Appl. Phys. Lett. 105, 132407 (2014).

${ }^{5}$ D. Zhu, M. Guizar-Sicairos, B. Wu, A. Scherz, Y. Acremann, T. Tyliszczak, P. Fischer, N. Friedenberger, K. Ollefs, M. Farle, J. R. Fienup, and J. Stöhr, Phys. Rev. Lett. 105, 043901 (2010).

${ }^{6}$ C. Moreno, C. Munuera, S. Valencia, F. Kronast, X. Obradors, and C. Ocal, Nano Lett. 10, 3828 (2010).

${ }^{7}$ D. Lu, D. J. Baek, S. S. Hong, L. F. Kourkoutis, Y. Hikita, and H. Y. Hwang, Nat. Mater. 15, 1255 (2016).

${ }^{8}$ S. Eisebitt, J. Lüning, W. F. Schlotter, O. Hellwig, W. Eberhardt, and J. Stöhr, Nature 432, 885 (2004)

${ }^{9}$ S. Eisebitt and W. Eberhardt, in Frontiers of Nano-Optoelectronic Systems, edited by L. Pavesi and E. Buzaneva (Springer-Science+Business Media BV, 2000), p. 347.

${ }^{10}$ N. Bergeard, S. Schaffert, V. López-Flores, N. Jaouen, J. Geilhufe, C. M. Günther, M. Schneider, C. Graves, T. Wang, B. Wu, A. Scherz, C. Baumier, R. Delaunay, F. Fortuna, M. Tortarolo, B. Tudu, O. Krupin, M. P. Minitti, J. Robinson, W. F. Schlotter, J. J. Turner, J. Lüning, S. Eisebitt, and C. Boeglin, Phys. Rev. B 91, 054416 (2015).

${ }^{11}$ A. Romano, J. Vanhellemont, H. Bender, and J. R. Morante, Ultramicroscopy 31, 183 (1989).

${ }^{12}$ S. Roberts, J. McCaffrey, L. Giannuzzi, S. Roberts, F. Stevie, and N. Zaluzec, in Progress in Transmission Electron Microscopy 1, edited by X.-F. Zhang and Z. Zhang (Springer International Publishing, Zürich, 2001), p. 301

${ }^{13}$ C. A. Volkert and A. M. Minor, MRS Bull. 32, 389 (2007).

${ }^{14}$ S. N. Piramanayagam, J. Appl. Phys. 102, 011301 (2007).

${ }^{15}$ D. Mitin, M. Grobis, and M. Albrecht, Rev. Sci. Instrum. 87, 023703 (2016).

${ }^{16}$ W. F. Schlotter, J. Lüning, R. Rick, K. Chen, A. Scherz, S. Eisebitt, C. M. Günther, W. Eberhardt, O. Hellwig, and J. Stöhr, Opt. Lett. 32, 3110 (2007).

${ }^{17}$ W. F. Schlotter, R. Rick, K. Chen, A. Scherz, J. Stöhr, J. Lüning, S. Eisebitt, C. M. Günther, W. Eberhardt, O. Hellwig, and I. McNulty, Appl. Phys. Lett. 89, 163112 (2006).

${ }^{18}$ J. Geilhufe, C. Tieg, B. Pfau, C. M. Günther, E. Guehrs, S. Schaffert, and S. Eisebitt, Opt. Express 22, 24959 (2014).

${ }^{19}$ B. Pfau and S. Eisebitt, in Synchrotron Light Sources and Free-Electron Lasers, edited by E. Jaeschke, S. Khan, J. Schneider, R. Jochen, and J. B. Hastings (Springer International Publishing, Zürich, 2015), p. 1093. 
${ }^{20}$ W. F. van Dorp and C. W. Hagen, J. Appl. Phys. 104, 081301 (2008).

${ }^{21}$ J. F. Ziegler, M. D. Ziegler, and J. P. Biersack, Nucl. Instrum. Methods Phys. Res., Sect. B 268, 1818 (2010).

${ }^{22}$ R. B. Laughlin, D. Pines, J. Schmalian, B. P. Stojkovic, and P. Wolynes, Proc. Natl. Acad. Sci. U. S. A. 97, 32 (2000).

${ }^{23}$ E. Dagotto, New J. Phys. 7, 67 (2005).

${ }^{24}$ E. Dagotto, Science 309, 257 (2005).

${ }^{25}$ M. M. Qazilbash, A. Tripathi, A. A. Schafgans, B. J. Kim, H. T. Kim, Z. Cai, M. V. Holt, J. M. Maser, F. Keilmann, O. G. Shpyrko, and D. N. Basov, Phys. Rev. B 83, 165108 (2011).

${ }^{26}$ I. Radu, K. Vahaplar, C. Stamm, T. Kachel, N. Pontius, H. A. Dürr, T. A. Ostler, J. Barker, R. F. L. Evans, R. W. Chantrell, A. Tsukamoto, A. Itoh, A. Kirilyuk, T. Rasing, and A. V. Kimel, Nature 472, 205 (2011).

${ }^{27}$ V. R. Morrison, R. P. Chatelain, K. L. Tiwari, A. Hendaoui, A. Bruhács, M. Chaker, and B. J. Siwick, Science 346, 445 (2014).

${ }^{28}$ G. Malinowski, F. Dalla Longa, J. H. H. Rietjens, P. V Paluskar, R. Huijink, H. J. M. Swagten, and B. Koopmans, Nat. Phys. 4, 855 (2008).

${ }^{29}$ E. Turgut, C. La-O-Vorakiat, J. M. Shaw, P. Grychtol, H. T. Nembach, D. Rudolf, R. Adam, M. Aeschlimann, C. M. Schneider, T. J. Silva, M. M. Murnane, H. C. Kapteyn, and S. Mathias, Phys. Rev. Lett. 110, 197201 (2013).

${ }^{30}$ C. E. Graves, A. H. Reid, T. Wang, B. Wu, S. de Jong, K. Vahaplar, I. Radu, D. P. Bernstein, M. Messerschmidt, L. Müller, R. Coffee, M. Bionta,
S. W. Epp, R. Hartmann, N. Kimmel, G. Hauser, A. Hartmann, P. Holl, H. Gorke, J. H. Mentink, A. Tsukamoto, A. Fognini, J. J. Turner, W. F. Schlotter, D. Rolles, H. Soltau, L. Strüder, Y. Acremann, A. V Kimel, A. Kirilyuk, T. Rasing, J. Stöhr, A. O. Scherz, and H. A. Dürr, Nat. Mater. 12, 293 (2013).

${ }^{31}$ T. Wang, D. Zhu, B. Wu, C. Graves, S. Schaffert, T. Rander, L. Müller, B. Vodungbo, C. Baumier, D. P. Bernstein, B. Bräuer, V. Cros, S. de Jong, R. Delaunay, A. Fognini, R. Kukreja, S. Lee, V. López-Flores, J. Mohanty, B. Pfau, H. Popescu, M. Sacchi, A. B. Sardinha, F. Sirotti, P. Zeitoun, M. Messerschmidt, J. Turner, W. F. Schlotter, O. Hellwig, R. Mattana, N. Jaouen, F. Fortuna, Y. Acremann, C. Gutt, H. A. Dürr, E. Beaurepaire, C. Boeglin, S. Eisebitt, G. Grübel, J. Lüning, J. Stöhr, and A. O. Scherz, Phys. Rev. Lett. 108, 267403 (2012).

${ }^{32}$ C. von Korff Schmising, B. Pfau, M. Schneider, C. M. Günther, M. Giovannella, J. Perron, B. Vodungbo, L. Müller, F. Capotondi, E. Pedersoli, N. Mahne, J. Lüning, and S. Eisebitt, Phys. Rev. Lett. 112, 217203 (2014).

${ }^{33}$ S. Schaffert, B. Pfau, J. Geilhufe, C. M. Günther, M. Schneider, C. von Korff Schmising, and S. Eisebitt, New J. Phys. 15, 093042 (2013).

${ }^{34}$ C.-J. Lai, K.-H. Hong, J. P. Siqueira, P. Krogen, C.-L. Chang, G. J. Stein, H. Liang, P. D. Keathley, G. Laurent, J. Moses, L. E. Zapata, and F. X. Kärtner, J. Opt. 17, 094009 (2015).

${ }^{35}$ S. M. Teichmann, F. Silva, S. L. Cousin, M. Hemmer, and J. Biegert, Nat. Commun. 7, 11493 (2016). 